\title{
Dielectric metasurface zone plate for the generation of focusing vortex beams
}

\author{
Yufeng $\mathrm{Hu}^{1 \dagger}{ }^{+}$, Xuan $\mathrm{Liu}^{2,3^{*}+}$, Mingke $\mathrm{Jin}^{4 \dagger}$, Yutao Tang ${ }^{4}$, Xuecai Zhang ${ }^{4}$, King Fai Li ${ }^{4}$, Yan Zhao ${ }^{2,3}$, Guixin $\mathrm{Li}^{4,5^{*}}$ and \\ Jing Zhou $^{1^{*}}$
}

\author{
* Correspondence: liuxuan@bjut. \\ edu.cn; ligx@sustech.edu.cn; jzhou@ \\ bnu.edu.cn \\ ${ }^{\dagger}$ Yufeng Hu, Xuan Liu and Mingke \\ Jin contributed equally to this work. \\ ${ }^{2}$ Institute of Laser Engineering, \\ Faculty of Materials and \\ Manufacturing, Beijing University of \\ Technology, Beijing 100124, China \\ ${ }^{4}$ Department of Materials Science \\ and Engineering, Southern \\ University of Science and \\ Technology, Shenzhen 518055 \\ China \\ ${ }^{1}$ Applied Optics Beijing Area Major \\ Laboratory, Department of Physics, \\ Beijing Normal University, Beijing \\ 100875, China \\ Full list of author information is \\ available at the end of the article
}

\begin{abstract}
Vortex beams carrying orbital angular momentum have important applications in high dimensional optical information processing, manipulations of tiny particles, super-resolution imaging and so on. Among various optical components, metasurface represents an ideal platform for realizing vortex beams with multiple optical functionalities due to its strong ability in manipulating the phase, polarization and amplitude of light. A metasurface combing the functions of a lens and a vortex beam generator can greatly shrink the size of many optical systems. Here, we alternatively propose a new metasurface design based on the concept of a Fresnel zone plate to generate, focus the vortex beams, and perform on-axis interference between different vortex beams. These functions are experimentally demonstrated through encoding the spiral phase profiles into the odd and even zones of a dielectric metasurface. The proposed vortex beam generation strategy employs the advantages of both the Fresnel zone plate and the metasurface, and may open new routes for high-dimensional optical information processing.
\end{abstract}

Keywords: Metasurface, Zone plate, Vortex beam

\section{Springer Open}

\section{Introduction}

In 1992, Allen et al. proposed that a Laguerre-Gaussian beam with a helical phasefront $\exp (i l \theta)$ carries orbital angular momentum (OAM) of $l$ h per photon [1], where $l$ is the topological charge which determines how fast the phase changes along the azimuthal angle $\theta, \hbar$ is the reduced Planck's constant. Since then, vortex beams (VBs) with OAM have attracted increasing attention for their various applications in particle manipulations [2, 3], high dimensional information processing [4-6], optical metrology [7, 8], etc. Although conventional devices including spiral phase plates [9], spatial light modulators [10] and so on have been widely used to generate optical VBs, however, they are usually bulky and thus limit the miniaturization of corresponding optical systems. To some extent, this constraint can be circumvented by using metasurfaces which are composed of spatially variant subwavelength meta-atoms [11]. In last few years, metasurface technologies have been successfully applied to realize various planar optical components, such as metalenses [12-15], holograms [16-22], vortex beam generators [23-35], pulse shaper [36] and so on. Recently, metasurface devices were also

(c) The Author(s). 2021 Open Access This article is licensed under a Creative Commons Attribution 4.0 International License, which permits use, sharing, adaptation, distribution and reproduction in any medium or format, as long as you give appropriate credit to the original author(s) and the source, provide a link to the Creative Commons licence, and indicate if changes were made. The images or other third party material in this article are included in the article's Creative Commons licence, unless indicated otherwise in a credit line to the material. If material is not included in the article's Creative Commons licence and your intended use is not permitted by statutory regulation or exceeds the permitted use, you will need to obtain permission directly from the copyright holder. To view a copy of this licence, visit http://creativecommons.org/licenses/by/4.0/. 
applied to the areas of quantum information. For example, metasurface chips for quantum entanglement states generation and reconstruction [37-39], cold atoms generation [40], high dimensional quantum source [41] were experimentally demonstrated.

Among various design strategies, the metasurfaces based on dynamic phase and geometric Pancharatnam-Berry (P-B) phase are usually used to design optical components with high efficiency. For dynamic phase metasurface [11, 14], the phase modulation at specific wavelength is usually implemented by changing the geometrical size of the meta-atoms. For the P-B phase metasurface [23], the spatially dependent phase of transmitted or reflected light can be achieved by changing the orientation angle of the anisotropic meta-atoms. The metasurface VB generators can directly impart a spiral phase factor $\exp (i l \theta)$ to the electric field of the incident light. In addition to generating VBs, metasurfaces are capable of integrating multiple functions into a single optical chip. For example, simultaneously generating and focusing of VBs were realized by using the phase profiles of a conventional lens [28-35]. A plasmonic metasurface VB generator working in the visible region has low optical efficiency [28]. In order to further improve optical efficiency, both all-dielectric [30] and the metal-dielectric hybrid [33-35] metasurfaces have been proposed. In the microwave regime, which is not the focus of this work, the generation of high efficiency VBs also attract quite a lot of attentions [27, 31, 32].

Like metasurfaces, the Fresnel zone plate $[42,43]$ is another kind of planar optical devices. By imparting the pre-defined surface profiles into the two sets of neighboring rings of the Fresnel zone plates, one can control the propagation of electromagnetic waves by using optical diffraction effect. In this work, it is expected that the metasurface based Fresnel zone plate can be used for generating, focusing VB and even preform other optical functions. In the proof of concept experiment, the dielectric metasurface (Fig. 1a), consisting of polarization-independent silicon nitride $\left(\mathrm{SiN}_{\mathrm{x}}\right)$ meta-atoms, are employed to generate the focusing VBs with OAM. In addition, we also demonstrated that the Hermite-Gaussian beam can be synthesized by generating two VBs with opposite OAM values and performing the on-axis interference. Compared to other strategies of generating the focusing multiple VBs, the metasurface zone plate proposed in this

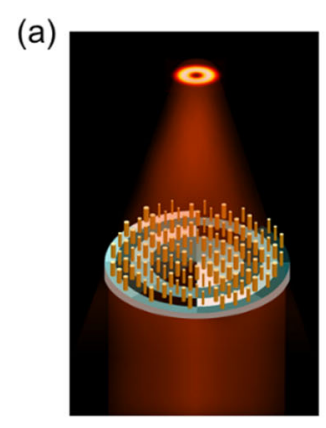

(b)

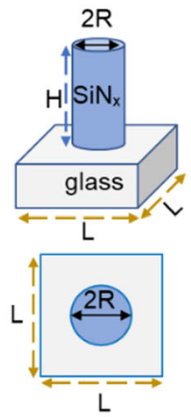

(c)

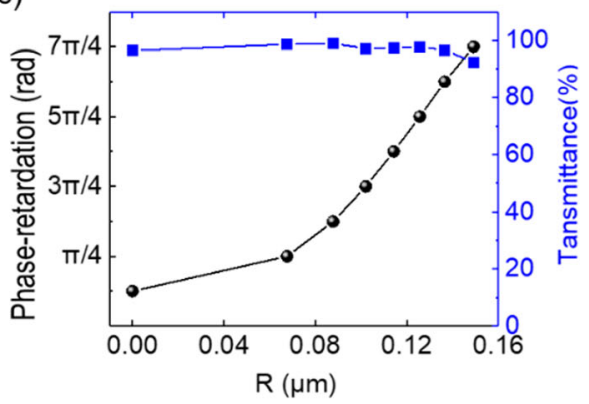

Fig. 1 Metasurface zone plate and the optical properties of the dielectric meta-atoms. a Schematic of a metasurface zone plate. $\mathbf{b}$ Side and top view of a single $\mathrm{SiN}_{\mathbf{x}}$ meta-atom, which is arranged in a square lattice. c The calculated phase-retardation and transmittance of a single meta-atom in a unit cell with $\mathrm{H}=$ $1 \mu \mathrm{m}, L=400 \mathrm{~nm}$ as a function of the radius $R$ at the incident light wavelength of $633 \mathrm{~nm}$ 
work represents a more intuitive route to introduce both the phase for focusing and the phase for controlling the topological charges of the vortex beams.

\section{Results and discussion}

\section{Design and fabrication of the metasurface zone plates}

According to the Huygens-Fresnel principle, a typical Fresnel zone plate for focusing light wave consists of concentric rings with radii of $r_{n}=\sqrt{n f \lambda_{0}+\frac{n^{2} \lambda_{0}^{2}}{4}}$, where $n$ is the serial number of rings, $\lambda_{0}$ is the wavelength of the incident light and $f$ is the focal length corresponding to $\lambda_{0}$ [42]. Under normal incidence, the lights transmitted from two neighbored rings should have a $\pi$-phase difference at the focal point. In other words, the lights transmitted from all the even-numbered zones or from all the odd-numbered zones have the phase difference of $2 q \pi$ at the focal point, $q$ is an arbitrary integer. In addition, the spiral phase zone plate for generating VB with specific OAM value can be obtained by introducing extra spiral phase profiles into the even- and odd-numbered zones respectively. Thus, the phase profiles of the spiral phase zone plate can be expressed as:

$$
\phi_{n}(\theta)=\left\{\begin{array}{cc}
l \theta, & n=2 m-1 \\
\pi+l \theta, & n=2 m
\end{array}\right.
$$

where $\theta=\arctan (y / x)$ represents the azimuth coordinate at any position $(x, y)$ on the zone plate and $m$ is nonzero positive integer.

It should be noted that, the odd- and even-numbered zones can also provide a degree of freedom for independently generating two VBs with different OAM values, and therefore can perform the on-axis interference between different VBs. In this case, the spiral phase profiles are defined as:

$$
\phi_{n}(\theta)=\left\{\begin{array}{lc}
l_{1} \theta, \quad n=2 m-1 \\
l_{2} \theta, \quad n=2 m
\end{array}\right.
$$

where $l_{1}$ and $l_{2}$ can be arbitrary integers, representing the topological charges of the VBs generated by the odd- and even-numbered zones, respectively.

In this work, the required phase profiles are introduced by using $\mathrm{SiN}_{\mathrm{x}}$ meta-atoms (Figs. 1a and b). The phase retardation, which is mainly due to the waveguide effect, can be described by $\phi_{W G}=\frac{2 \pi}{\lambda} n_{\text {eff }} H$, where $n_{\text {eff }}$ and $H$ are the effective index and the height of the meta-atoms [14]. At the wavelength of $633 \mathrm{~nm}$, the values of $H$ and $L$ are numerically optimized, which are $H=1 \mu \mathrm{m}$ and $L=400 \mathrm{~nm}$. Then, the value of $n_{\text {eff }}$ can be adjusted by varying the radius of the meta-atom. Then, the phase retardations of the meta-atoms with different radii are calculated by using commercial finite difference time domain (FDTD) solver (Lumerical Inc.). The measured complex refractive index of the $\mathrm{SiN}_{\mathrm{x}}$ material (Fig. S1) is used in the calculation. As shown in Fig. 1c, the eight phase retardations are equally spaced ranging from zero to $2 \pi$. The transmittances of the meta-atoms in a periodic lattice are also calculated and all of them are above $90 \%$.

To verify the concept of generating focusing VB with the metasurface zone plate, four metasurface devices with eight phase steps are designed and fabricated. The focal lengths $f$ of all the devices are set to be $2.0 \mathrm{~mm}$ at the wavelength of $633 \mathrm{~nm}$. Figures 2a and $\mathrm{b}$ show the phase profiles for generating focusing VBs with topological charges of 




Fig. 2 Design and fabrication of the dielectric metasurface zone plates. a-d The calculated phase profiles of the metasurface zone plates with topological charges of $(\mathbf{a}) I=2,(\mathbf{b}) I=3,(\mathbf{c}) I=+/-2$, (d) $I=+/-3$. e-h The top-view SEM images of the fabricated metasurfaces corresponding to (a-d). Scale bar: $20 \mu \mathrm{m}$. $\mathbf{i}-\mathbf{I}$ The side-view SEM images corresponding to (e-h). Scale bar: 1 um

$l=2$ and 3, respectively. In comparison, Figs. $2 \mathrm{c}$ and $\mathrm{d}$ correspond to the metasurfaces for generating focusing VBs with topological charges of $+/-2$ and $+/-3$, respectively. The scanning electron microscopic (SEM) images of the fabricated samples are shown in Figs. 2e-1, where all the areas without meta-atoms correspond to the phase retardation of zero.

\section{Optical characterization of the fabricated metasurface zone plates}

All the four fabricated metasurface zone plates are experimentally characterized by using a home-build optical setup (Fig. S2). Firstly, the focusing properties of the fabricated metasurface zone plates with topological charges of $l=2$ and $l=3$ are measured. According to the geometrical symmetry of the meta-atom, we know that these samples are insensitive to the polarization of normally incident light. Without lack of generality, Figs. 3a-d and i-l show the measured intensity profiles along the propagating direction with horizontally polarized incident lights at four different wavelengths of $633 \mathrm{~nm}, 600$ $\mathrm{nm}, 570 \mathrm{~nm}$, and $532 \mathrm{~nm}$. The corresponding focal lengths at the four wavelengths are $2.01 \mathrm{~mm}, 2.12 \mathrm{~mm}, 2.23 \mathrm{~mm}$, and $2.37 \mathrm{~mm}$, respectively. This negative dispersion property of the focal length is consistent with the theoretical prediction. According to the diffraction theory, the focal length of a Fresnel zone plate is $f(\lambda)=\frac{r_{1}^{2}}{\lambda}$ [42], where $\lambda$ is the incident wavelength and $r_{1}$ is the radius of the first zone. The calculated relative deviations of the four measured focal lengths from the theoretical values are all less than $1 \%$.

Figures $3 \mathrm{e}-\mathrm{h}$ and $\mathrm{m}-\mathrm{p}$ show the doughnut-shaped intensity profiles of light at the corresponding focal planes at the four wavelengths of the metasurfaces with topological charges of $l=2$ and 3 . The intensity distributions at the focal planes can be analytically calculated by using Kirchhoff-Fresnel diffraction integral formula (Fig. S5), which are consistent with the experimental results. In order to determine the orbital angular momentum of the vortex beams, we calculate the off-axis interference patterns of the 
(a)
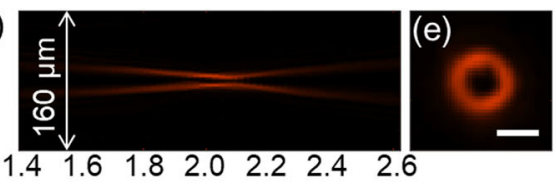

(b)
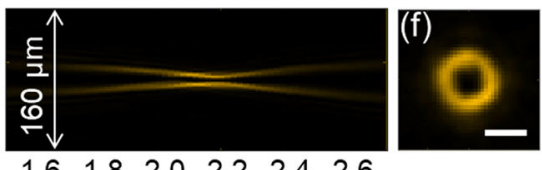

(c)
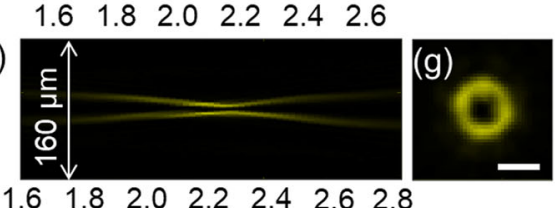

(d)
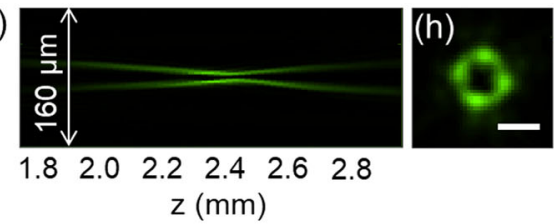
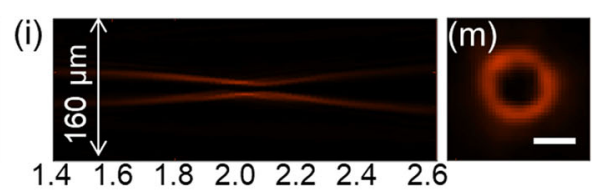

(j)
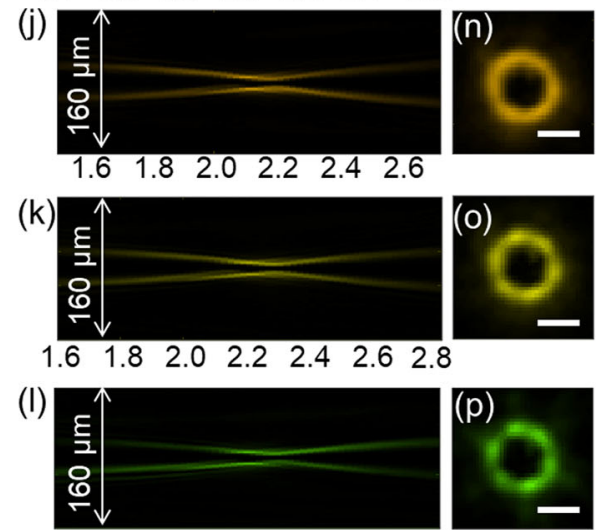

$\begin{array}{llllll}1.8 & 2.0 & 2.2 & 2.4 & 2.6 & 2.8\end{array}$

$\mathrm{z}(\mathrm{mm})$

Fig. 3 The measured intensity profiles of the VBs along the propagating direction for the metasurface zone plates with topological charges of $(\mathbf{a}-\mathbf{d}) I=2$ and $(\mathbf{i}-\mathbf{I}) I=3$ at different wavelengths of $(\mathbf{a}, \mathbf{i}) 633 \mathrm{~nm},(\mathbf{b}, \mathbf{j})$ $600 \mathrm{~nm},(\mathbf{c}, \mathbf{k}) 570 \mathrm{~nm}$, and $(\mathbf{d}, \mathbf{I}) 532 \mathrm{~nm}$. e-h and $\mathbf{m}-\mathbf{p}$ are measured intensity profiles at the focal planes corresponding to (a-d) and (i-l). Scale bar: $10 \mu \mathrm{m}$. All the images are shown in false-color

vortex beams with a Gaussian beam. As shown in Fig. S6, the fork patterns have two and three dislocated fringes, which indicate that topological charges of the VBs are $l=2$ and $l=3$, respectively. The intensity profiles in both Figs. 3e-h and Figs. 3m-p gradually deviate from an ideal doughnut shape when the wavelength of light is away from the designed wavelength of $633 \mathrm{~nm}$. This is because that both the phase retardation and the transmission efficiency of every single $\operatorname{SiN}_{\mathrm{x}}$ metaatom are away from the optimized values. In order to verify the polarization insensitivity of our design, vertically polarized incident light is also used in the experiment. The measured results are same as those using a horizontally polarized incident light (Fig. S3). In addition, the polarization state of the transmitted light is experimentally analyzed (Figs. $4 \mathrm{a}$ and $\mathrm{b}$ ). The polarization state of the transmitted light is almost same as that of the incident light.


Fig. 4 The measured intensity profiles at the focal planes of the metasurface device with topological charge of $I=2$ at wavelength of $633 \mathrm{~nm}$. a H-H polarization configuration. $\mathbf{b} \mathrm{H}-\mathrm{V}$ polarization configuration. c The measured optical efficiencies of the metasurface devices with topological charges of $I=2$ (red lines) and $I=3$ (blue lines). 'H-H', 'H-V' represent the horizontally polarized incident light with a horizontally or vertically polarized analyzer for the transmitted wave 
Under the illumination of horizontally polarized light, the optical efficiencies of the metasurfaces with topological charges of $l=2$ and $l=3$ are characterized. As shown in Fig. 4c, the focusing efficiencies of the two metasurface devices are above $12 \%$ over the wavelength region from $560 \mathrm{~nm}$ to $680 \mathrm{~nm}$. For the two metasurface devices, there are some differences in the wavelength dependent diffraction efficiency. This should be due to the different spiral phase profiles encoded into the metasurface zone plates. It seems that the measured optical efficiencies of the metasurfaces deviates far from the calculated ones in Fig. 1b. However, it should be noted that the meta-atoms in Fig. 1b are periodically arranged in a square lattice, which is very different from the metasurface zone plate. To estimate the diffraction efficiency of the metasurfaces, we assume the metasurface zone plate has an ideal phase distribution as that of a conventional Fresnel zone plate. The theoretical focusing efficiency of the primary focal point of an ideal phase-type zone plate is about $40.5 \%$ (Supplementary Information Section V). Thus, it reasonable to obtain the measured focusing efficiency lower than this value. We expect that the measured focusing efficiencies of the metasurface zone plate can be improved by optimizing the nanofabrication processes.

The metasurface devices with composite topological charges of $l_{1}=-l_{2}=2$ and $l_{1}=$ $-l_{2}=3$ are also experimentally characterized. The intensity distributions of the VBs at the focal planes are shown in Figs. 5a-d, with working wavelengths of $633 \mathrm{~nm}, 600 \mathrm{~nm}, 570$ $\mathrm{nm}$, and $532 \mathrm{~nm}$ respectively. The petal distributions are produced by the interference of two different VBs. Comparing with the theoretically calculated results in Figs. 5e and j, it is found that the experimental results are consistent with the theoretical expectations. By using similar concepts, on-axis interference between arbitrary two VBs can be realized by encoding the spiral phase profiles into the odd- and even-numbered zones of a dielectric metasurface. This proposed device may have important applications in quantum information processing [37-39], angular velocity measurement of an object, rotating a tiny particle and so on.

\section{Conclusions}

In summary, the designs of polarization-insensitive metasurface zone plates for generating the focusing VB and the multiple VBs with different OAM values are proposed and experimentally demonstrated. The meta-surface zone plate takes the advantages of the



Fig. 5 The measured intensity profiles at the focal planes of the metasurface devices with topological charges of $(\mathbf{a}-\mathbf{d}) /=+/-2$ and $(\mathbf{f}-\mathbf{i}) /=+/-3$. The light wavelengths are $(\mathbf{a}, \mathbf{f}) 633 \mathrm{~nm},(\mathbf{b}, \mathbf{g}) 600 \mathrm{~nm},(\mathbf{c}, \mathbf{h})$ $570 \mathrm{~nm}$, and (d, i) $532 \mathrm{~nm}$, respectively. The images are shown in false-color. Scale bar:10 $\mu \mathrm{m}$. e, j The calculated intensity profiles corresponding to that in $(\mathbf{a}, \mathbf{f})$ 
intuitive design of the conventional phase-type Fresnel zone plate and the multiple degrees of freedom of the metasurfaces. It should be noted that the numerical aperture of the metasurface zone plate and topological charges of the generated vortex beams will finally be limited by the pixel size of the meta-atoms. The proposed strategy in this work may open new avenues for designing optical vortex beams with multiple functionalities.

\section{Methods}

\section{Nanofabrication of the metasurfaces}

Firstly, a $1000 \mathrm{~nm}$ thick $\mathrm{SiN}_{\mathrm{x}}$ film was deposited on a silica substrate by using plasma enhanced chemical vapor deposition method. Then, a $125 \mathrm{~nm}$ thick electron resist (PMMA) layer was spin-coated onto the substrate and baked at the temperature $180^{\circ} \mathrm{C}$ for 3 min. After that, a charge-dissipation layer was spin-coated on top of the PMMA layer and baked at the temperature of $90^{\circ} \mathrm{C}$ for $2 \mathrm{~min}$. Subsequently, the patterns of the metasurfaces were written into the PMMA layer by using the electron beam lithography. The charge-dissipation layer was removed with DI water, and the PMMA layer was developed with MIBK: IPA solution for two mins. Afterwards, a $20 \mathrm{~nm}$ thick $\mathrm{Cr}$ layer was deposited on top of the PMMA pattern by using e-beam evaporation and the patterns are transferred to the Cr layer through lift-off processes. The sample with the patterned $\mathrm{Cr}$ hard mask layer was etched through the inductively-coupled-plasma etching process. Finally, the metasurface devices were obtained after the removal of the $\mathrm{Cr}$ layer in the chromium etchant solution.

\footnotetext{
Abbreviations

VB: Vortex beam; OAM: Orbital angular momentum; P-B: Pancharatnam-Berry; SiN ${ }_{x}$ : Silicon nitride; FDTD: Finite difference time domain; SEM: Scanning electron microscope
}

\section{Supplementary Information}

\section{The online version contains supplementary material available at https://doi.org/10.1186/s43074-021-00035-z.}

Additional file 1. This supplementary information provides details on refractive index of the $\mathrm{SiN}_{\mathrm{x}}$ layer, the optical measurements and the calculated intensity distributions of the vortex beams.

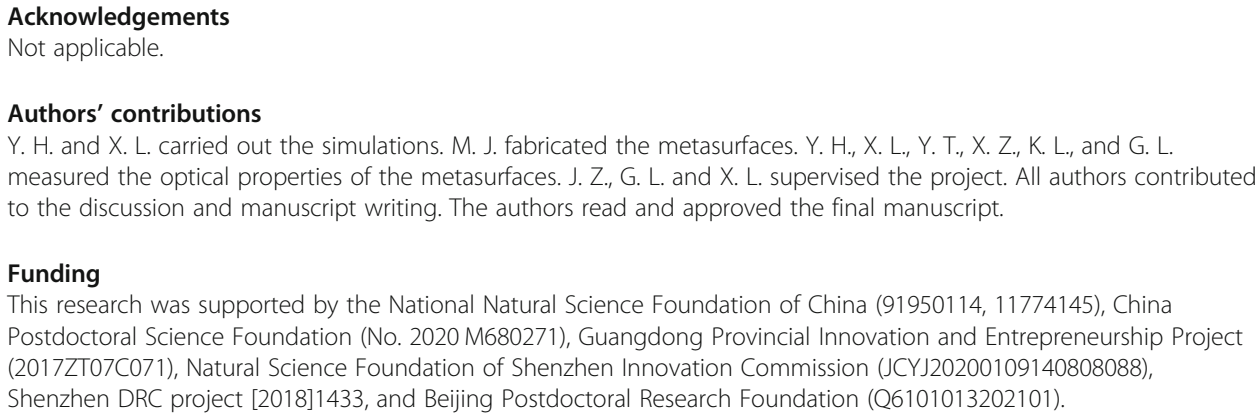

This research was supported by the National Natural Science Foundation of China (91950114, 11774145), China Postdoctoral Science Foundation (No. 2020 M680271), Guangdong Provincial Innovation and Entrepreneurship Project (2017ZT07C071), Natural Science Foundation of Shenzhen Innovation Commission (JCYJ20200109140808088), Shenzhen DRC project [2018]1433, and Beijing Postdoctoral Research Foundation (Q6101013202101).

Availability of data and materials

The data and the relevant methods are available on request from the corresponding authors.

\section{Declarations}

Ethics approval and consent to participate

Not applicable. 


\section{Author details}

${ }^{1}$ Applied Optics Beijing Area Major Laboratory, Department of Physics, Beijing Normal University, Beijing 100875, China. ${ }^{2}$ Institute of Laser Engineering, Faculty of Materials and Manufacturing, Beijing University of Technology, Beijing 100124, China. ${ }^{3}$ Key Laboratory of Trans-scale Laser Manufacturing Technology, Beijing University of Technology, Ministry of Education, Beijing 100124, China. ${ }^{4}$ Department of Materials Science and Engineering, Southern University of Science and Technology, Shenzhen 518055, China. ${ }^{5}$ Shenzhen Engineering Research Center for Novel Electronic Information Materials and Devices, Southern University of Science and Technology, Shenzhen 518055, China.

Received: 27 April 2021 Accepted: 11 June 2021

Published online: 23 June 2021

\section{References}

1. Allen L, Beijersbergen MW, Spreeuw R, Woerdman JP. Orbital angular momentum of light and the transformation of Laguerre-Gaussian laser modes. Phys Rev A. 1992;45:8185-9.

2. Simpson NB, Allen L, Padgett MJ. Optical tweezers and optical spanners with Laguerre-Gaussian modes. J Mod Opt. 1996:43:2485-91.

3. Grier DG. A revolution in optical manipulation. Nature. 2003:424:810-6.

4. Gibson G, Courtial J, Padgett MJ, Vasnetsov M, Pas'ko V, Barnett SM, et al. Free-space information transfer using light beams carrying orbital angular momentum. Opt Express. 2004;12:5448-56.

5. Mair A, Vaziri A, Weihs G, Zeilinger A. Entanglement of the orbital angular momentum states of photons. Nature. 2001; 412:313-6.

6. Vallone G, D'Ambrosio V, Sponselli A, Slussarenko S, Marrucci L, Sciarrino F, et al. Free-space quantum key distribution by rotation-invariant twisted photons. Phys Rev Lett. 2014;113:060503.

7. Lavery MP, Speirits FC, Barnett SM, Padgett MJ. Detection of a spinning object using light's orbital angular momentum. Science. 2013;341:537-40.

8. Georgi P, Schlickriede C, Li G, Zhang S, Zentgraf T. Rotational Doppler shift induced by spin-orbit coupling of light at spinning metasurfaces. Optica. 2017:4:1000-5.

9. Beijersbergen M, Coerwinkel R, Kristensen M, Woerdman J. Helical wavefront laser beams produced with a spiral phase plate. Opt Commun. 1994;112:321-7.

10. Leach J, Gibson GM, Padgett MJ, Esposito E, McConnell G, Wright AJ, et al. Generation of achromatic Bessel beams using a compensated spatial light modulator. Opt Express. 2006;14:5581-7.

11. Yu N, Genevet P, Kats MA, Aieta F, Tetienne JP, Capasso F, et al. Light propagation with phase discontinuities: generalized laws of reflection and refraction. Science. 2011;334:333-7.

12. Khorasaninejad M, Chen WT, Devlin RC, Oh J, Zhu AY, Capasso F. Metalenses at visible wavelengths: diffraction-limited focusing and subwavelength resolution imaging. Science. 2016;352:1190-4.

13. Khorasaninejad M, Capasso F. Metalenses: Versatile multifunctional photonic components. Science. 2017;358:eaam8100.

14. Khorasaninejad M, Zhu AY, Roques-Carmes C, Chen WT, Oh J, Mishra I, et al. Polarization-insensitive metalenses at visible wavelengths. Nano Lett. 2016;16:7229-34.

15. Balli F, Sultan M, Lami SK, Hastings JT. A hybrid achromatic metalens. Nat Commun. 2020;11:1-8.

16. Zheng G, Müuhlenbernd H, Kenney M, Li G, Zentgraf T, Zhang S. Metasurface holograms reaching $80 \%$ efficiency. Nat Nanotechnol. 2015;10:308-12.

17. Wang L, Kruk S, Tang H, Li T, Kravchenko I, Neshev DN, et al. Grayscale transparent metasurface holograms. Optica. 2016: 3:1504-5.

18. Deng Z-L, Li G. Metasurface optical holography. Mater Today Phys. 2017:3:16-32.

19. Mao N, Deng J, Zhang $X$, Tang Y, Jin M, Li Y, et al. Nonlinear diatomic metasurface for real and Fourier space image encoding. Nano Lett. 2020;20:7463-8.

20. Ding X, Wang Z, Hu G, Liu J, Zhang K, Li H, et al. Metasurface holographic image projection based on mathematical properties of Fourier transform. PhotoniX. 2021;1:16.

21. Chen WT, Yang K-Y, Wang C-M, Huang Y-W, Sun G, Chiang I-D, et al. High-efficiency broadband meta-hologram with polarization-controlled dual images. Nano Lett. 2014;14:225-30.

22. Wen D, Yue F, Li G, Zheng G, Chan K, Chen S, et al. Helicity multiplexed broadband metasurface hologram. Nat Commun. 2015;6:8241.

23. Li G, Kang M, Chen S, Zhang S, Pun EYB, Cheah KW, et al. Spin-enabled plasmonic metasurfaces for manipulating orbital angular momentum of light. Nano Lett. 2013;13:4148-51.

24. Maguid E, Yulevich I, Veksler D, Kleiner V, Brongersma ML, Hasman E. Photonic spin-controlled multifunctional sharedaperture antenna array. Science. 2016;352:1202-6.

25. Devlin RC, Ambrosio A, Rubin NA, Mueller JB, Capasso F. Arbitrary spin-to-orbital angular momentum conversion of light. Science. 2017:358:896-901.

26. Yuan $Y$, Zhang K, Ratni B, Song Q, Ding X, Wu Q, et al. Independent phase modulation for quadruplex polarization channels enabled by chirality-assisted geometric-phase metasurfaces. Nat Commun. 2020;11:4186.

27. Yuan Y, Sun S, Chen Y, Zhang K, Ding X, Ratni B, et al. A fully phase-modulated metasurface as an energy-controllable circular polarization router. Adv Sci. 2020;7:2001437.

28. Mehmood MQ, Mei S, Hussain S, Huang K, Siew SY, Zhang L, et al. Visible-frequency metasurface for structuring and spatially multiplexing optical vortices. Adv Mater. 2016;28:2533-9.

29. Ma X, Pu M, Li X, Huang C, Wang Y, Pan W, et al. A planar chiral meta-surface for optical vortex generation and focusing. Sci Rep. 2015;5:10365.

30. Ou K, Li G, Li T, Yang H, Yu F, Chen J, et al. High efficiency focusing vortex generation and detection with polarizationinsensitive dielectric metasurfaces. Nanoscale. 2018;10:19154-61.

31. Bai X, Kong F, Qian J, Song Y, He C, Liang X, et al. Polarization-insensitive metasurface lens for efficient generation of convergent OAM beams. IEEE Antennas and Wireless Propagation Lett. 2019;18:2696-700. 
32. Zhang K, Yuan Y, Zhang D, Ding X, Ratni B, Burokur SN, et al. Phase-engineered metalenses to generate converging and non-diffractive vortex beam carrying orbital angular momentum in microwave region. Opt Express. 2018;26:1351-60.

33. Liu X, Deng J, Jin M, Tang Y, Zhang X, Li KF, et al. Cassegrain metasurface for generation of orbital angular momentum of light. Appl Phys Lett. 2019;115:221102.

34. Ding F, Chen Y, Bozhevolnyi SI. Focused vortex-beam generation using gap-surface plasmon metasurfaces. Nanophotonics. 2020;9:371-8.

35. Tang S, Ding F. High-efficiency focused optical vortex generation with geometric gap-surface plasmon metalenses. Appl Phys Lett. 2020;117:011103.

36. Divitt S, Zhu W, Zhang C, Lezec HJ, Agrawal A. Ultrafast optical pulse shaping using dielectric metasurfaces. Science. 2019;364:890-4.

37. Wang K, Titchener JG, Kruk SS, Xu L, Chung H-P, Parry M, et al. Quantum metasurface for multiphoton interference and state reconstruction. Science. 2018;361:1104-8.

38. Stav T, Faerman A, Maguid E, Oren D, Kleiner V, Hasman E, et al. Quantum entanglement of the spin and orbital angular momentum of photons using metamaterials. Science. 2018;361:1101-4.

39. Georgi P, Massaro M, Luo K-H, Sain B, Montaut N, Herrmann H, et al. Metasurface interferometry toward quantum sensors. Light Sci Appl. 2019;8:70.

40. Zhu L, Liu X, Sain B, Wang M, Schlickriede C, Tang Y, et al. Dielectric metasurface optical chip for the generation of cold atoms. Sci Adv. 2020;6:eabb6667.

41. Li L, Liu Z, Ren X, Wang S, Su V-C, Chen M-K, et al. Metalens-array-based high-dimensional and multiphoton quantum source. Science. 2020;368:1487-90.

42. Attwood D. Soft X-Rays and Extreme Ultraviolet Radiation: Principles and Applications. Cambridge: Cambridge University Press; 1999.

43. Born M, Wolf E. Principles of optics. Cambridge: Cambridge University Press; 1980.

\section{Publisher's Note}

Springer Nature remains neutral with regard to jurisdictional claims in published maps and institutional affiliations.

\section{Submit your manuscript to a SpringerOpen ${ }^{\circ}$ journal and benefit from:}

- Convenient online submission

Rigorous peer review

- Open access: articles freely available online

- High visibility within the field

- Retaining the copyright to your article

Submit your next manuscript at $\boldsymbol{\nabla}$ springeropen.com 